\title{
Privacy-Preserving Mining of Sequential Association Rules from Provenance Workflows
}

\author{
Mihai Maruseac \\ UMass Boston \\ mmarusea@cs.umb.edu
}

\author{
Gabriel Ghinita \\ UMass Boston \\ gabriel.ghinita@umb.edu
}

\begin{abstract}
Provenance workflows capture movement and transformation of data in complex environments, such as document management in large organizations, content generation and sharing in in social media, scientific computations, etc. Sharing and processing of provenance workflows brings numerous benefits, e.g., improving productivity in an organization, understanding social media interaction patterns, etc. However, directly sharing provenance may also disclose sensitive information such as confidential business practices, or private details about participants in a social network. We propose an algorithm that privately extracts sequential association rules from provenance workflow datasets. Finding such rules has numerous practical applications, such as capacity planning or identifying hot-spots in provenance graphs. Our approach provides good accuracy and strong privacy, by leveraging on the exponential mechanism of differential privacy. We propose an heuristic that identifies promising candidate rules and makes judicious use of the privacy budget. Experimental results show that the our approach is fast and accurate, and clearly outperforms the state-of-the-art. We also identify influential factors in improving accuracy, which helps in choosing promising directions for future improvement.
\end{abstract}

\section{INTRODUCTION}

Provenance workflows are increasingly important in characterizing industrial processes, scientific computations, social media interactions and content generation. Provenance is vital in understanding and optimizing processes involved with complex data management tasks. In recent years, numerous research efforts focused on capturing and analyzing the information provided by provenance workflows. A survey of existing techniques and important research directions in studying provenance can be found in [5]. Sharing provenance brings numerous benefits. For example, chemists can exchange process data for synthesizing new drugs. Corporations may better integrate operations with business partners.

Consider the example in Figure 1, which shows several document flows between departments in a company. Even though most documents would follow the path Production to Accounting and then Sales, some might flow through Audit, Compliance and

Permission to make digital or hard copies of part or all of this work for personal or classroom use is granted without fee provided that copies are not made or distributed for profit or commercial advantage and that copies bear this notice and the full citation on the first page. Copyrights for third-party components of this work must be honored. For all other uses, contact the owner/author(s).

CODASPY'16 March 09-11, 2016, New Orleans, LA, USA

(C) 2016 Copyright held by the owner/author(s).

ACM ISBN 978-1-4503-3935-3/16/03.

DOI: http: //dx.doi.org/10.1145/2857705.2857743

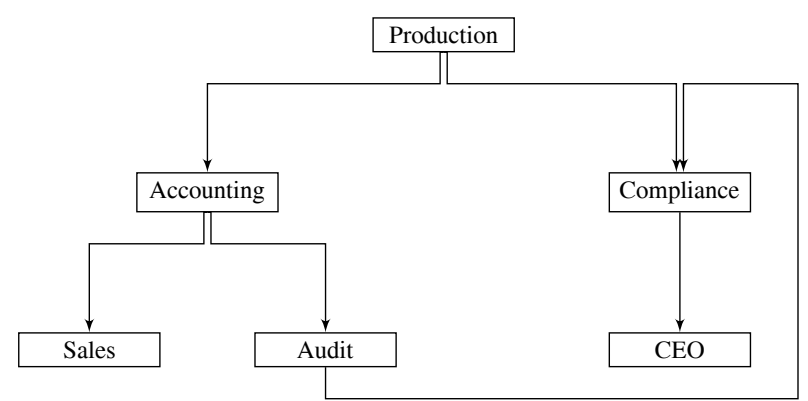

Figure 1: Document Workflows in an Organization

then $C E O$. The existence of such a document might reveal a highrisk event (e.g., a compliance oversight) which might impact the organization's future if publicly released. The objective of sanitizing provenance is to prevent an adversary from identifying such high-risk events, while at the same time allowing researchers to extract useful aggregate-level information from the provenance.

The differential privacy (DP) [1] framework is designed specifically for such aggregate-level release of information, and it is widely accepted as the de-facto standard for privacy-preserving data publication. DP preserves aggregate information, but it prevents an adversary from inferring whether any particular data item was present in the input dataset or not. For example, it would be impossible to detect if a certain secret chemical element was used in synthesizing a specific drug. Several techniques for rule mining with differential privacy have been proposed, culminating with the work in [3], which outperforms other existing techniques. However, such work focuses on itemset mining, and ignores sequence information, which is essential for provenance processing. None of the existing approaches we are aware of focus on provenance data, which is more challenging due to high sparsity.

In this paper, we focus on privately extracting sequential association rules from provenance data. We use the exponential mechanism of differential privacy, and introduce a powerful selection heuristic that identifies promising rules to sample. Experimental results show that our approach is fast, and it clearly outperforms existing techniques with respect to accuracy of extracting sequential association rules from provenance data.

\section{PRELIMINARIES}

Provenance workflows. Consider an undirected graph $G=$ $(V, E)$, where each node $x_{i} \in V$ represents an entity which generates, receives or forwards data (e.g., department of an organization, user of a social network, etc.). The set $E$ of edges captures the connections between these entities. Nodes are labeled with natural numbers, that is $V \subset \mathbb{N}$ and $E \subset \mathbb{N}^{2}$. A provenance workflow is a path in graph $G$, allowing a node to be visited multiple times to capture provenance loops. A path is a sequence $\pi_{i}=x_{1}^{(i)} \rightarrow x_{2}^{(i)} \rightarrow$ 


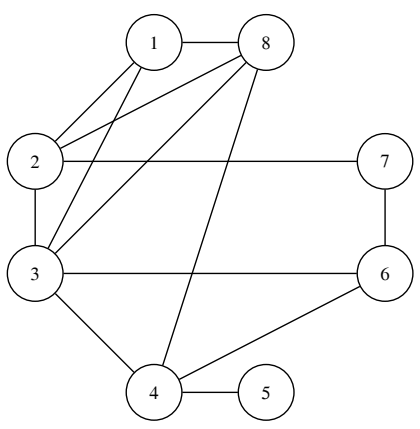

(a) Running example graph

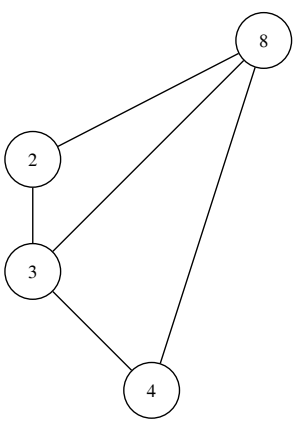

(b) Set $S_{\text {initial }}$

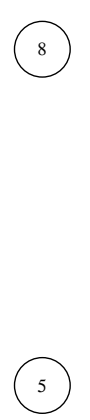

(c) Set $S_{b a d}$

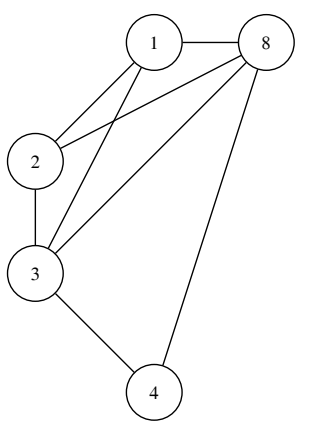

(d) BFS approach

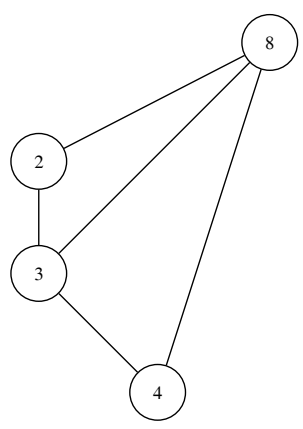

(e) Truncated BFS

Figure 2: Running example graph (a); sets $S_{i}$ generated with the algorithm in [3] (b,c) and with our approach (d, e)

\begin{tabular}{|l|r|r|}
\hline Node & Real Count & Noisy Count \\
\hline 4 & 100 & 142 \\
\hline 3 & 90 & 86 \\
\hline 2 & 63 & 70 \\
\hline 8 & 67 & 65 \\
\hline 7 & 50 & 52 \\
\hline 6 & 34 & 30 \\
\hline 5 & 25 & 29 \\
\hline 1 & 10 & 5 \\
\hline
\end{tabular}

Table 1: Real and noisy node counts (sorted by noisy count) $\ldots x_{t_{i}}^{(i)}$ where each $x_{i} \in V$ is a node in $G, t_{i}$ is the length of the path $\pi_{i}$ and there is an edge between any two consecutive nodes in the path. In Figure 1, $\pi_{1}=$ Production $\rightarrow$ Accounting $\rightarrow$ Sales and $\pi_{2}=$ Production $\rightarrow$ Accounting $\rightarrow$ Audit $\rightarrow$ Compliance $\rightarrow C E O$ are workflows.

Differential privacy (DP) [1] guarantees that for any two sibling datasets $\mathcal{D}_{1}, \mathcal{D}_{2}$ that differ in a single workflow $\pi$, the probability of an adversary learning which of the two datasets was used to obtain a certain output $\mathcal{A}$ is bounded by $\left|\ln \frac{\operatorname{Pr}\left[\mathcal{A}\left(\mathcal{D}_{1}\right)\right]}{\operatorname{Pr}\left[\mathcal{A}\left(\mathcal{D}_{2}\right)\right]}\right| \leq \epsilon$, where parameter $\epsilon>0$ represents the privacy budget. To achieve privacy for numerical queries, the Laplace mechanism adds to each query result noise randomly distributed according to a Laplace distribution with parameter $\lambda=S / \epsilon$ where $S$ is the sensitivity of the query, i.e., the maximum change in the result of the query for any two sibling databases. On the other hand, the exponential mechanism [4] allows to privately sample data, by associating each possible output $o$ a weight $q(o)$, where $q$ is a quality function. Formally, each possible output $o$ is selected with probability proportional to $\exp \left(\frac{\epsilon q(o)}{2 S}\right)$ where $S$ is the sensitivity of $q$.

\section{PROPOSED APPROACH}

A sequential association rule is an implication $X \Rightarrow Y$ where $X$ and $Y$ are fragments of a path in $G$. We define a path fragment as a sequence $X=x_{1} \rightarrow x_{2} \rightarrow \ldots \rightarrow x_{l_{X}}$ of length $l_{X}$ where nodes $x_{1} \ldots x_{l_{X}} \in V$ are linked by edges in $E$. For each path fragment $X$, we associate a metric called support, denoted by $\sigma(X)$, which is the number of workflows $\pi_{i}$ in the database which contain the sequence $X$. The intuition of an association rule $X \Rightarrow Y$ is that a provenance workflow which contains $X$ also contains $Y$ and that the path fragment $X$ is followed by the path fragment $Y$ (in other words, the workflow also contains the path fragment $X \rightarrow Y$ ). The confidence of a rule is defined as $c(X \Rightarrow Y)=\frac{\sigma(X \rightarrow Y)}{\sigma(X)}$. Our objective is to privately extract high-confidence sequential association rules from the provenance database. One distinguishing characteristic of our work in comparison with existing techniques (e.g., [3]) is that we take into account sequence information, whereas other approaches are concerned with frequent itemsets, regardless of the order of appearance of items. Specifically, our algorithm must be able to analyze both the rule $1 \rightarrow 2 \Rightarrow 3$ and $2 \rightarrow 1 \Rightarrow 3$ whereas for existing approaches ([3]) these rules look identical.

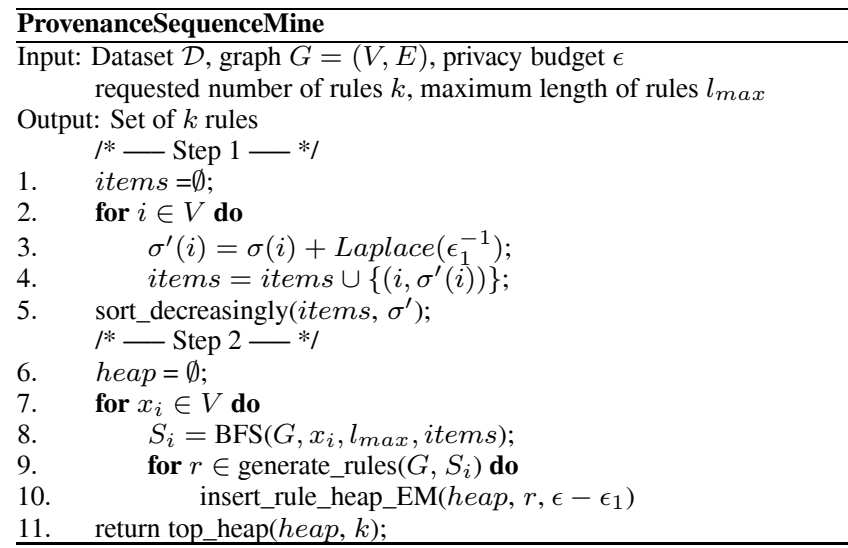

Figure 3: Pseudocode of Two-Step Proposed Approach

The proposed algorithm has two steps, summarized by the pseudocode in Figure 3. The first step (lines 1-5) uses a small fraction of the privacy budget, $\epsilon_{1}=\alpha \times \epsilon$, to compute basic statistics on the input database. For each node, we query the total number of times any document has passed through that node in order to obtain an estimation of the hotspots in the workflows. We protect these counts with the Laplace mechanism. For example, Figure 2(a) illustrates a provenance workflow graph, and Table 1 captures statistics for a set of workflows. For instance, node 4 has been visited by a total of 100 documents (i.e., real count of workflows) and after Laplace noise addition the sanitized count becomes 142 .

The main contribution of the algorithm lies within the second step. From the list of noisy counts obtained in Step 1, we select sets $S_{i}$ of cardinality $l_{\max }$ nodes from which we generate association rules of lengths up to $l_{\max }$. Next, from all sets $S_{i}$, we use the exponential mechanism to select the $k$ rules with the highest (noisy) confidence. Heap operations follow the framework of reservoir sampling [2] to allow for an efficient sampling of rules by trading some memory space to reduce time complexity.

Central to our approach is the choice of quality function $q$ used within the exponential mechanism (EM), which must capture well the confidence of a rule, but at the same time must preserve privacy. In addition, it is important to devise an effective heuristic to prune the total number of candidate rules considered by EM, as the total number of candidates grows exponentially, but only relatively few candidates yield high-confidence rules. To illustrate the importance of these factors, we first show the limitations of the approach in [3] which does not account for provenance graph structure. In [3], a 


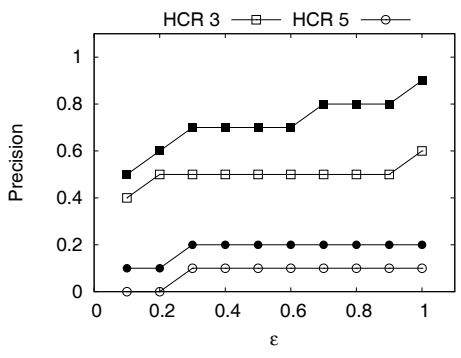

(a) $k=10$

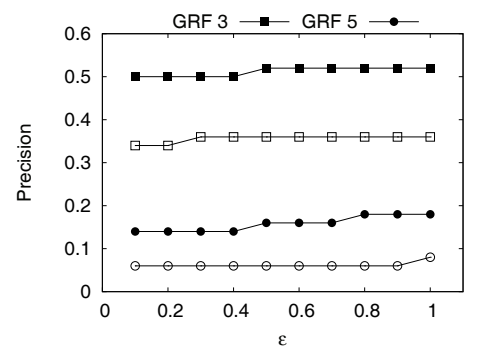

(b) $k=50$

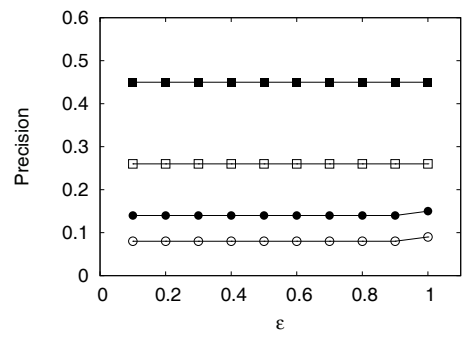

(c) $k=100$

Figure 4: Accuracy of the proposed approach in comparison with benchmark [3]

sliding window of size $l_{\max }$ is constructed, with noisy counts from Step 1 in descending order. For example, in Figure 2(a), assume $l_{\max }=4$. The approach in [3] would generate rules with items from the set $S_{\text {initial }}=\{4,3,2,8\}$ (the most 4 frequent elements in the workflows) making sure that there is always a path between the nodes in the rule. Hence, it can generate the rule $8 \rightarrow 2 \Rightarrow 3$ but it cannot generate rule $8 \rightarrow 2 \Rightarrow 4$ because there is no edge $(2,4)$ in the subgraph spanned by the set $S_{\text {initial }}$, as seen in Figure 2(b).

However, as [3] does not account for graph structure, a future position of the sliding window will generate the set $S_{b a d}=$ $\{8,7,6,5\}$ which generates the subgraph shown in Figure 2(c). This graph has only a single edge $(6,7)$, hence it can only generate rules involving repetitions of the two nodes linked by it, for example $6 \Rightarrow 7$. It is likely that from set $S_{b a d}$ we can only generate rules with poor quality. If such sets are abundant, which is the case if we use the sliding window approach, the majority of rules inserted in the heap will have bad quality, increasing the chances of sampling bad rules and reducing the accuracy of the algorithm.

In contrast, we use the graph structure to select nodes from which to generate candidate rules. Any workflow which passes through a frequent node will also pass through at least one of its neighbors. It is more likely for a frequent node to occur in the middle of several intersecting workflows. Hence, we propose to generate the sets $S_{i}$ by considering nodes $x_{i} \in V$ in descending order of the noisy counts from Step 1. For each $x_{i}$ we add to the set $S_{i}$, we also add the nodes obtained in a breadth-first search (BFS) traversal of the graph starting at $x_{i}$. We truncate the search when at least $l_{\max }$ elements are found in $S_{i}$. In the running example, for node 8 , we obtain set $S_{8}=\{8,1,2,3,4\}$ with the corresponding subgraph shown in Figure 2(d), after the first level of the BFS traversal.

This example also shows another limitation: even though we are guaranteed to always generate connected subgraphs, their size can grow far larger than $l_{\text {max }}$. For any such set $S_{i}$ we have that $\left|S_{i}\right| \geq l_{\max }$ so we can generate rules of lengths up to $l_{\max }$. However, there are $\left(\begin{array}{l}\left|S_{i}\right| \\ l_{\max }\end{array}\right)$ ways to select the $l_{\max }$ items from $S_{i}$, leading to an exponential blow-up in the candidate generation phase of the algorithm. To address this issue, we devise a strategy that stops the BFS traversal as soon as $l_{\max }$ items have been inserted into the set $S_{i}$, thus transforming the search from a truncated BFS to an iterative deepening approach. Because this will most likely interrupt the traversal in the middle of a level, we need to prioritize the order in which the neighbors of a node are visited. Specifically, we visit the nodes in decreasing order of the noisy counts from Step 1.

The pseudocode of Step 2 is captured in lines 6-10 of Figure 3. Note that, Step 2 only makes use of the relative ordering of graph nodes, and not their associated noisy counts. Hence, the privacy budget allocated to the first step can be fairly small, dedicating most of $\epsilon$ to rule sampling in Step 2.

\section{EXPERIMENTAL EVALUATION}

We implemented a $\mathrm{C}$ prototype of the proposed technique, and we use as benchmark the method from [3]. We consider a workflow database modeled as a graph with 10 nodes and 50 edges on top of which we generate 10,000 provenance documents with path lengths uniformly distributed between 5 and 7 . The experiments were run on an Intel Core i7-3770 $3.4 \mathrm{GHz}$ CPU machine with 8 cores and $16 \mathrm{~GB}$ of RAM, running Linux OS. As evaluation metric, we consider the precision of returned rules, i.e., the fraction of rules with confidence above a threshold $c_{0}=0.4$ (we omit other thresholds due to space limitations). We extract between $k=10$ and $k=100$ sequences with lengths between $l_{\max }=3$ and $l_{\max }=5$. In all runs, the execution time was always below three seconds.

Figure 4 (a)-(c) shows that our graph-centric approach (label GRF) consistently outperforms the benchmark from [3] (label HCR). For the lowest number of extracted rules $k=10$, our method reaches precision of $100 \%$ for larger $\varepsilon$ values. As the length of the rules grows, the accuracy of both methods decreases due to the combinatorial increase in the number of candidate rules. However, our method is still able to outperform the benchmark, and is less adversely affected by the increase of $l_{\max }$.

As expected, both methods are negatively impacted by the increase in $k$, as the privacy budget needs to be divided across more invocations of the exponential mechanism. Our method still outperforms the benchmark, but further research is necessary to identify ways to increase precision for larger $k$.

\section{CONCLUSION}

We proposed a novel method to mine sequence association rules from provenance workflows with differential privacy. Experimental results show that the approach is able to effectively make use of provenance graph information to improve mining accuracy, and clearly outperforms existing techniques. In future work, we will investigate methods to further enhance precision by using more information from the structure of the graph and by considering alternative privacy budget allocation strategies.

Acknowledgments. This work has been supported by NSF award CNS-1111512.

\section{REFERENCES}

[1] C. Dwork, F. McSherry, K. Nissim, and A. Smith. Calibrating noise to sensitivity in private data analysis. In TCC, pages 265-284, 2006.

[2] M. Kolonko and D. Wäsch. Sequential reservoir sampling with a nonuniform distribution. ACM Trans. Math. Softw., 32(2):257-273, June 2006.

[3] M. Maruseac and G. Ghinita. Differentially-Private Mining of High-Confidence Association Rules. Proc. of CODASPY, 2015.

[4] F. McSherry and K. Talwar. Mechanism design via differential privacy. In Proc. of IEEE Symp. on Foundations of Computer Science, pages 94-103, 2007.

[5] Y. L. Simmhan, B. Plale, and D. Gannon. A survey of data provenance in e-science. SIGMOD Rec., 34(3):31-36, Sept. 2005. 